\title{
THE TOTAL GRAPH OF A COMMUTATIVE SEMIRING
}

\author{
Shahabaddin Ebrahimi Atani and Fatemeh Esmaeili Khalil Saraei
}

\begin{abstract}
We introduce and investigate the total graph of a commutative semiring with non-zero identity. The main purpose of this paper is to extend the definition and some results given in [2] to a more general semiring case.
\end{abstract}

\section{Introduction}

The concepts of the graph of the zero-divisors of a ring was first introduced by Beck in [5] when discussing the coloring of a commutative ring. In his work all elements of the ring were vertices of the graph. D. D. Anderson and Naseer use this same concept in [1]. We adopt the approach used by D.F. Anderson and Livingston in [3] and consider only nonzero zero-divisors as vertices of the graph. D.F. Anderson and Livingston, and Mulay in [13] examined, among other things, the diameter and girth of the zero-divisor graph of a commutative ring. Let $R$ be a commutative ring with $Z(R)$ its set of zero-divisors elements. The total graph of $R$, denoted by $T(\Gamma(R)$ ), is the (undirected) graph with all elements of $R$ as vertices, and for distinct $x, y \in R$, the vertices $x$ and $y$ are adjacent if and only if $x+y \in Z(R)$. The total graph of a commutative ring have been introduced and studied by D.F. Anderson and A. Badawi in [2]. In [10], the notion of the total torsion element graph of a module over a commutative ring is introduced. Some other investigations into properties of zero-divisor graph of a commutative semiring may be found in $[7,8]$.

Key Words: Semiring, total graph, $k$-ideal, $Q$-ideal.

2010 Mathematics Subject Classification: 16Y60,05C75.

Received: July 2011.

Revised: February 2012.

Accepted: June 2012. 
Ideals of semirings play a central role in the structure theory and are useful for many purposes $[11,12]$. However, they do not in general coincide with the ideals of rings and, for this reason, their use is somewhat limited in trying to obtain analogues of ring theorems for semirings. Indeed, many results in rings apparently have no analogues in semirings using only ideals. In order to overcome this deficiency, the authors defined a more restricted class of ideals in semirings, which are called the class of " $k$-ideals" and the class of " $Q$-ideals" $[4,11,12,6]$. In the present paper we introduce a new class of graphs, called the total graph of a commutative semiring, and we completely characterize the structure of this graph. The total graph of a commutative ring and the total graph of a commutative semiring are different concepts. Some of our results are analogous to the results given in [2]. The corresponding results are obtained by modification and here we give a complete description of the total graph of a commutative semiring. The study of the total graph of a commutative semiring $R$ breaks naturally into two cases depending on whether or not $Z(R)$ is an ideal of $R$. In the third section, we handle the case when $Z(R)$ is not an ideal of $R$; in the fourth section, we do the case when $Z(R)$ is an ideal of $R$ ((either $k$-ideal or $Q$-ideal $)$ ).

\section{Preliminaries}

For the sake of completeness, we state some definitions and notations used throughout. For a graph $\Gamma$, by $E(\Gamma)$ and $V(\Gamma)$, we denote the set of all edges and vertices, respectively. We recall that a graph is connected if there exists a path connecting any two distinct vertices. At the other extreme, we say that a graph is totally disconnected if no two vertices of this graph are adjacent. The distance between two distinct vertices $a$ and $b$, denoted by $\mathrm{d}(a, b)$, is the length of a shortest path connecting them (if such a path does not exist, then $d(a, a)=0$ and $d(a, b)=\infty)$. The diameter of a graph $\Gamma$, denoted by $\operatorname{diam}(\Gamma)$, is equal to $\sup \{d(a, b): a, b \in V(\Gamma)\}$. A graph is complete if it is connected with diameter less than or equal to one. The girth of a graph $\Gamma$, denoted $\operatorname{gr}(\Gamma)$, is the length of a shortest cycle in $\Gamma$, provided $\Gamma$ contains a cycle; otherwise; $\operatorname{gr}(\Gamma)=\infty$. We denote the complete graph on $n$ vertices by $K^{n}$ and the complete bipartite graph on $m$ and $n$ vertices by $K^{m, n}$ (we allow $m$ and $n$ to be infinite cardinals). We will sometimes call a $K^{1, m}$ a star graph. We say that two (induced) subgraphs $\Gamma_{1}$ and $\Gamma_{2}$ of $\Gamma$ are disjoint if $\Gamma_{1}$ and $\Gamma_{2}$ have no common vertices and no vertex of $\Gamma_{1}$ (respectively, $\Gamma_{2}$ ) is adjacent (in $\Gamma$ ) to any vertex not in $\Gamma_{1}$ (respectively, $\Gamma_{2}$ ).

Throughout this paper $R$ is a commutative semiring with identity. In order to make this paper easier to follow, we recall in this section various notions

from semiring theory which will be used in the sequel. For the definitions of 
monoid, semirings, and ideals we refer $[11,12,4,6]$. All semiring in this paper are commutative with non-zero identity. Let $R$ be a semiring.

(1) A semiring $R$ is said to be a semidomain whenever $a, b \in R$ with $a b=0$ implies that either $a=0$ or $b=0$.

(2) A subtractive ideal (=k-ideal) $I$ is an ideal of $R$ such that if $x, x+y \in I$, then $y \in I$ (so $\left\{0_{R}\right\}$ is a $k$-ideal of $R$ ).

(3) An element $x$ of $R$ is called a zero-sum in $R$ if $x+y=0$ for some $y \in R$. We use $S(R)$ to denote the set of all zero-sum elements of $R$.

(4) A semiring $R$ is called a $R$-cancellative semiring if whenever $r s=r t$ for elements $s, t, r \in R$ with $r \neq 0$, then $s=t$.

(5) An ideal $I$ of a semiring $R$ is called a partitioning ideal $(=Q$-ideal $)$ if there exists a subset $Q$ of $R$ such that

(a) $R=\cup\{q+I: q \in Q\}$

(b) If $q_{1}, q_{2} \in Q$, then $\left(q_{1}+I\right) \cap\left(q_{2}+I\right) \neq \emptyset$ if and only if $q_{1}=q_{2}$.

Let $I$ be a $Q$-ideal of $R$ and let $R / I=\{q+I: q \in Q\}$. Then $R / I$ forms a semiring under the operations $\oplus$ and $\odot$ defined as follows: $\left(q_{1}+I\right) \oplus\left(q_{2}+I\right)=$ $q_{3}+I$, where $q_{3} \in Q$ is the unique element such that $q_{1}+q_{2}+I \subseteq q_{3}+I$ and $\left(q_{1}+I\right) \odot\left(q_{2}+I\right)=q_{4}+I$, where $q_{4} \in Q$ is the unique element such that $q_{1} q_{2}+I \subseteq q_{4}+I$. This semiring $R / I$ is called the quotient semiring of $R$ by $I[5]$.

(6) We define the total graph of a semiring $R$, denoted by $T(\Gamma(R))$, as follows: $V(T(\Gamma(R)))=R, E(T(\Gamma(R)))=\{\{x, y\}: x+y \in Z(R)\}$. We will use $\operatorname{Reg}(R)$ to denote the set of elements of $R$ that are not zero-divisors. Let $\operatorname{Reg}(\Gamma(R))$ be the (induced) subgraph of $T(\Gamma(R))$ with vertices $\operatorname{Reg}(R)$, and let $\mathrm{Z}(\Gamma(R))$ be the (induced) subgraph of $T(\Gamma(R))$ with vertices $Z(R)$.

\section{$3 Z(R)$ is not an ideal of $R$}

Let $R$ be a commutative ring. In this section, we study the total graph $T(\Gamma(R))$ when $Z(R)$ is not an ideal of $R$. Our stating point is the following proposition:

Proposition 3.1. Let $R$ be a commutative semiring. Then the following hold:

(i) If $R$ is a semi-domain with $2=1_{R}+1_{R}=\{0\}$, then $R$ is a ring.

(ii) If $r \in \operatorname{Reg}(R)$, then $2 \in Z(R)$ if and only if $2 r \in Z(R)$.

Proof. (i) Let $r \in R$. We may assume that $r \neq 0$. By assumption, there exists $0 \neq s \in R$ such that $2 s=0$. Since $s(2 r)=(2 s) r=0$, we have $2 r \in Z(R)=\{0\}$, as required.

(ii) It suffices to show that if $2 r \in Z(R)$, then $2 \in Z(R)$. There exists a non-zero element $s$ of $R$ such that $(2 s) r=s(2 r)=0$; hence $2 s=0$ since 
$r \notin Z(R)$. Thus $2 \in Z(R)$.

Theorem 3.2. Let $R$ be a commutative semiring. Then the following hold:

(i) $T(\Gamma(R))$ is complete if and only if $Z(R)=R$.

(ii) $T(\Gamma(R))$ is totally disconnected if and only if $Z(R)=S(R)=\{0\}$.

Proof. (i) The sufficiency is clear. Conversely, suppose that $T(\Gamma(R))$ is complete and let $r \in R$. Then $r$ is adjacent to 0 . Thus $r=r+0 \in Z(R)$, and hence we have equality.

(ii) Let $T(\Gamma(R))$ be totally disconnected. Then 0 is not adjacent to any vertex; hence $r=r+0 \notin Z(R)$ for every non-zero element $r$ of $R$. Thus $Z(R)=\{0\}$. If there exists a non-zero element $s$ of $S(R)$, then there is an element $0 \neq t \in R$ such that $s+t=0 \in Z(R)$, which is a contradiction. Thus $S(R)=\{0\}$. Conversely, suppose that there exist distinct $a, b \in R$ such that $a+b \in Z(R)=\{0\}$. Then $a, b \in S(R)$, a contradiction. Hence $T(\Gamma(R))$ is totally disconnected.

Lemma 3.3. Let $R$ be a semiring such that $Z(R)$ is not an ideal of $R$. Then there are distinct $r, r^{\prime} \in Z(R)^{*}$ such that $r+r^{\prime} \in \operatorname{Reg}(R)$.

Proof. It is enough to show that $Z(R)$ is always closed under scalar multiplication of its elements by elements of $R$. Let $a \in Z(R)$ and $r \in R$. There is a non-zero element $s \in R$ with $s a=0$; hence $s(r a)=r(s a)=0$. Thus $r a \in Z(R)$. This completes the proof.

Theorem 3.4. Let $R$ be a semiring such that $Z(R)$ is not an ideal of $R$. Then $\mathrm{Z}(\Gamma(R))$ is connected with $\operatorname{diam}(\mathrm{Z}(\Gamma(R)))=2$.

Proof. Let $r \in Z(R)^{*}$. Then $r$ is adjacent to 0 . Thus $r-0-s$ is a path in $\mathrm{Z}(\Gamma(R))$ of length two between any two distinct $r, s \in Z(R)^{*}$. Moreover, there exist nonadjacent $r, s \in Z(R)^{*}$ by Lemma 3.3; thus $\operatorname{diam}(\mathrm{Z}(\Gamma(R)))=2$.

Example 3.5 shows that Theorem 3.1 (2) and Theorem 3.3 in [2], in general, are not true when $R$ is a semiring.

Example 3.5 Let $S=\{0,1, a\}$ be the idempotent semiring in which $1+a=a+1=a$ and let $R=S \oplus S$. Then $R$ is a semiring with 9 elements. An inspection will show that $Z(R)=\{(0,0),(1,0),(0,1),(a, 0),(0, a)\}$ is not an ideal of $R$ and $R=\langle Z(R)\rangle$. Moreover, $Z(\Gamma(R))$ is disjoint from $\operatorname{Reg}(\Gamma(R))$ and $\operatorname{Reg}(\Gamma(R))$ is a totally disconnected subgraph of $T(\Gamma(R))$. Hence $T(\Gamma(R))$ is disconnected.

Definition 3.6. A semiring $R$ is called a subtractive semiring if every cyclic ideal of $R$ is a k-ideal. 
Example 3.7. Assume that $E_{+}$be the set of all non-negative integers and let $R=E_{+} \cup\{\infty\}$. Define $a+b=\max \{a, b\}$ and $a b=\min \{a, b\}$ for all $a, b \in R$. Then $R$ is a commutative semiring with $1_{R}=\infty$ and $0_{R}=0$. An inspection will show that the list of ideals of $R$ are: $R, E_{+}$and for every non-negative integer $n$

$$
I_{n}=\{0,1, \ldots, n\} .
$$

It is clear that every proper ideal of $R$ is a $k$-ideal. So $R$ is a subtractive semiring.

lemma 3.8. Let $R$ be a subtractive semiring which is not a ring. Then $S(R) \subseteq Z(R)$.

Proof. If $S(R)=\{0\}$, we are done. Suppose that $0 \neq r \in S(R)$. Then there is a $s \in S(R)$ such that $r+s=0$. Thus $s \in R r$ since $R r$ is a $k$-ideal. Then there exists $t \in R$ such that $(1+t) r=0$. It then follows from [9, Lemma 2.1] that $1+t \neq 0$. Thus $r \in Z(R)$, as required.

Theorem 3.9. Let $R$ be a subtractive semiring which is not a ring. If $|S(R)| \geq 3$, then $\operatorname{gr}(Z(\Gamma(R)))=3$.

Proof. By assumption and Lemma 3.8, there are non-zero elements $x, y$ of $S(R)$ with $x, y \in Z(R)$ and $x+y \in Z(R)$. Thus $0-x-y-0$ is a 3 -cycle in $Z(\Gamma(R))$, as required.

Theorem 3.10. Let $R$ be a semiring $R$ such that $Z(R)$ is not an ideal of $R$. Then either $\operatorname{gr}(Z(\Gamma(R)))=3$ or $\operatorname{gr}(Z(\Gamma(R)))=\infty$.

Proof. If $x+y \in Z(R)$ for some distinct $x, y \in Z(R)^{*}$, then $0-x-y-0$ is a 3-cycle in $Z(\Gamma(R))$; so $\operatorname{gr}(Z(\Gamma(R)))=3$. Otherwise, $x+y \in \operatorname{Reg}(R)$ for all distinct $x, y \in Z(R)$. Therefore, in this case, each $x \in Z(R)^{*}$ is adjacent to 0 , and no two distinct $x, y \in Z(R)^{*}$ are adjacent. Thus $Z(\Gamma(R))$ is a star graph with center 0 ; hence $\operatorname{gr}(Z(\Gamma(R)))=\infty$.

Lemma 3.11. Let $R$ be a semiring $R$ such that $Z(R)$ is not an ideal of $R$. Then $|Z(R)| \geq 3$.

Proof. By Lemma 3.3, there are distinct $x, y \in Z(R)^{*}$ such that $x+y \in$ $\operatorname{Reg}(R)$; hence $|Z(R)| \geq 3$.

Theorem 3.12. Let $R$ be a semiring such that $Z(R)$ is not an ideal of $R$. Then $\operatorname{gr}(\operatorname{Reg}(\Gamma(R)))=3$ or $\infty$. 
Proof. We may assume that $\operatorname{Reg}(\Gamma(R))$ contains a cycle. So there is a path $x-y-z$ in $\operatorname{Reg}(R)$. If $x+z \in Z(R)$, then we have a 3-cycle in $\operatorname{Reg}(\Gamma(R))$. So we may assume that $x+z \notin Z(R)$. There exist $r_{1}, r_{2} \in Z(R)$ such that $r_{1}+r_{2} \notin Z(R)$ by Lemma 3.3. So there are $0 \neq t_{1}, t_{2} \in R$ such that $r_{1} t_{1}=$ $r_{2} t_{2}=0$ and then $t_{1} t_{2}=0$ since $t_{1} t_{2}\left(r_{1}+r_{2}\right)=0$. Therefore $t_{1} x+t_{1} z \in Z(R)$ since $t_{2}\left(t_{1} x+t_{1} z\right)=0$. Thus $t_{1} x-t_{1} y-t_{1} z-t_{1} x$ is a 3 -cycle in $\operatorname{Reg}(\Gamma(R))$ and the proof is complete.

\section{$4 \quad Z(R)$ is an ideal of $R$}

Let $R$ be a commutative semiring. The structure of the total graph $T(\Gamma(R))$ may be completely described in those cases when zero-divisor elements form an ideal.

Proposition 4.1. Let $R$ be a commutative semiring $R$ such that $Z(R)$ is an ideal of $R$. Then the following hold:

(i) $Z(\Gamma(R))$ is a complete (induced) subgraph of $T(\Gamma(R))$.

(ii) If $I$ is an ideal of $R$, then $T(\Gamma(I))$ is an induced subgraph of $T(\Gamma(R))$ if and only if $Z(I)=I \cap Z(R)$.

(iii) If $(0: R) \neq 0$, then $T(\Gamma(R))$ is a complete graph.

Proof. The proofs are straightforward.

Example 4.2. (1) An ideal of a semiring in general need not be a either $k$-ideal or $Q$-ideal. Let $R$ be the set of all real numbers $x$ satisfying $0<x \leq 1$, and define $a+b=a . b=\min \{a, b\}$ for all $a, b \in R$. Then $(R,+,$.$) is easily$ checked to be a commutative semiring with 1 as identity. Each real number $r$ such that $0<r<1$ defines an ideal $I_{r}=\{t \in R: t \leq r\}$ of $R$. However, $r+1=r$ together $r \in I_{r}$ and $1 \notin I_{r}$ show that $I_{r}$ is not a $k$-ideal of $R$. In particular, $I_{r}$ is not a $Q$-ideal of $R$ since every $Q$-ideal is a $k$-ideal.

(2) Let $R$ denote the semiring of non-negative integers with the usual operations of addition and multiplication. If $m \in R-\{0\}$, the ideal

$$
I_{m}=\{k m: k \in R\}
$$

is a $Q$-ideal of $R$ when $Q=\{0,1, \cdots, m-1\}$. In particular, $I_{m}$ is a $k$-ideal.

(3) Assume that $R$ denote the semiring of non-negative integers. Define $x+y=\operatorname{gcd}(x, y)$ and $x . y=\operatorname{lcm}(\mathrm{x}, \mathrm{y})$. It is easy to see that $R$ is a semiring in which every element is idempotent. The ideal $I=\{0,2,4, \cdots\}$ is a $k$-ideal of $R$ but is not a $Q$-ideal.

Remark 4.3. Let $R$ be a semidomain. Then $Z(R)=\{0\}$ is a $Q$-ideal of $R$, where $Q=R-\{0\}$ (so it is a $k$-ideal of $R$ ). 
Example 4.4 shows that there is a commutative semiring $R$ such that $Z(R)$ is an ideal of $R$, but it is not a $k$-ideal.

Example 4.4. Assume that $E_{+}$be the set of all non-negative integers and let $R=\left\{(a, b): a, b \in E_{+}\right\}$. Define $(a, b)+(c, d)=(\min \{a, c\}, \max \{b, d\})$ and $(a, b) *(c, d)=(a c, b d)$ for all $(a, b),(c, d) \in R$. Then $(R,+, *)$ is easily checked to be a commutative semiring. An inspection will show that $Z(R)=\{(a, b) \in$ $R: a=0$ or $b=0\}$ is an ideal of $R$. However, $(0,1)+(2,5)=(0,5) \in Z(R)$ together with $(2,5) \notin Z(R)$ and $(0,1) \in Z(R)$ show that $Z(R)$ is not a $k$-ideal of $R$. Also, $T(\Gamma(R))$ is a connected graph since every element is adjacent to $(0,0)$ in $T(\Gamma(R))$. Moreover, $\operatorname{gr}(T(\Gamma(R)))=3$ since there is a 3 -cyclic $(0,0)-(0,1)-(1,0)-(0,0)$ in $T(\Gamma(R))$.

Example 4.5 shows that there is a commutative semiring such that $Z(R)$ is a $k$-ideal but it is not $Q$-ideal.

Example 4.5. Assume that $R$ is the set of all non-negative integers and let $a, b, k \in R$. Define $a+b=\operatorname{gcd}(a, b)$ and

$$
a * b= \begin{cases}0 & \text { if } \operatorname{gcd}(a, b)=2 k \\ 1 & \text { if } \operatorname{gcd}(a, b)=2 k+1 \\ 0 & \text { if } a=0 \text { or } b=0\end{cases}
$$

Then $(R,+, *)$ is easily checked to be a commutative semiring which is not a semidomain (note that $2 * 6=0$ ). An inspection will show that $Z(R)=$ $\{0,2,4,6, \cdots\}$ is a $k$-ideal of $R$ but is not a $Q$-ideal of $R$ by Example $4.2(3)$. Moreover, $Z(\Gamma(R))$ is a complete graph and $\operatorname{Reg}(\Gamma(R))$ is a totally disconnected graph.

The main goal of this section is a general structure theorem (Theorem 4.8) for $\operatorname{Reg}(\Gamma(R))$ when either $Z(R)$ is a $k$-ideal of $R$ or $Z(R)$ is a $Q$-ideal. But first, we record the basic observation that if $Z(R)$ is a $k$-ideal of (resp. $Z(R)$ is not a $k$-ideal), then the subgraph $Z(\Gamma(R))$ is disjoint from $\operatorname{Reg}(\Gamma(R))$ (resp. $Z(\Gamma(R))$ is not disjoint from $\operatorname{Reg}(\Gamma(R))$. Thus we will concentrate on the subgraph $\operatorname{Reg}(\Gamma(R))$ throughout this section.

Theorem 4.6. Let $R$ be a commutative semiring such that $Z(R)$ is a $k$-ideal of $R$. If $r$ and $r^{\prime}$ are distinct elements of $\operatorname{Reg}(R)$ that are connected by a path with $r+r^{\prime} \notin Z(R)$ (i.e., if $r$ and $r^{\prime}$ are not adjacent), then there is a path in $\operatorname{Reg}(\Gamma(M))$ of length at most 2 between $r$ and $r^{\prime}$.

Proof. Let $Z(R)$ be a $k$-ideal of $R$. It suffices to show that if $r_{1}, r_{2}, r_{3}$ and $r_{4}$ are distinct vertices of $\operatorname{Reg}(R)$ and there is a path $r_{1}-r_{2}-r_{3}-r_{4}$ from $r_{1}$ to $r_{4}$, then $r_{1}$ and $r_{4}$ are adjacent. Now we have $r_{1}+r_{2}+r_{3}+r_{4} \in Z(R)$. Then $Z(R)$ being $k$-ideal of $R$ gives $r_{1}+r_{4} \in Z(R)$, and so $r_{1}$ and $r_{4}$ are adjacent, as required. 
Compare the next theorem with [2, Theorem 2.1].

Theorem 4.7. Let $R$ be a commutative semiring $R$. Then the following hold:

(i) If $Z(R)$ is a $k$-ideal of $R$, then $Z(\Gamma(R))$ is disjoint from $\operatorname{Reg}(\Gamma(R))$.

(ii) If $Z(R)$ is not a $k$-ideal of $R$, then $Z(\Gamma(R))$ is not disjoint from $\operatorname{Reg}(\Gamma(R))$.

Proof. (i) If $Z(\Gamma(R))$ is not disjoint from $\operatorname{Reg}(\Gamma(R))$, then there exist $r \in Z(R)$ and $s \in \operatorname{Reg}(R)$ such that $r+s \in Z(R)$. Thus $s \in Z(R)$ since $Z(R)$ is a $k$-ideal of $R$ which is a contradiction. Thus $Z(\Gamma(R))$ is disjoint from $\operatorname{Reg}(\Gamma(R))$.

(ii) By assumption, there exist $a \in Z(R)$ and $b \in \operatorname{Reg}(R)$ such that $a+b \in$ $Z(R)$. Let $x \in R$. We define the subset $N(x)$ as follows: $N(x)=$

$$
\{r \in Z(R): \text { there is a path of finite length between } x \text { and } r\} .
$$

It is clear that if $x \in Z(R)$, then $Z(R) \subseteq N(x)$, and so $N(x) \neq \emptyset$. Set $I=\{x \in$ $R: N(x) \neq \emptyset\}$. Therefore, $Z(R) \subset I$ since $b \in I$ and $b \notin Z(R)$. Now we show that $I$ is an ideal of $R$. Let $r_{1}, s_{1} \in I$. Therefore, there exist $t_{1}, t_{1}^{\prime} \in Z(R)$, $r_{1}, r_{2}, \cdots, r_{n} \in R$ and $s_{1}, s_{2}, \cdots, s_{k} \in R$ such that $r_{1}-r_{2}-\cdots-r_{n}-t_{1}$ and $s_{1}-s_{2}-\cdots-s_{k}-t_{1}^{\prime}$ are paths of finite lengths between $r_{1}, t_{1}$ and $s_{1}, t_{1}^{\prime}$, and so we have $r_{i}+r_{i+1}, s_{j}+s_{j+1}, r_{n}+t_{1}, s_{k}+t_{1}^{\prime}, t_{1}+t_{1}^{\prime} \in Z(R)$ for each $1 \leq i \leq n-1$ and $1 \leq j \leq k-1$. We may assume that $n \leq k$. So $\left(r_{i}+s_{i}\right)+\left(r_{i+1}+s_{i+1}\right) \in$ $Z(R)$ for each $1 \leq i \leq n-1$. Then $\left(r_{1}+s_{1}\right)-\left(r_{2}+s_{2}\right)-\cdots-\left(r_{n}+s_{n}\right)-$

$$
\left(t_{1}+s_{n+1}\right)-\left(t_{1}^{\prime}+s_{n+2}\right)-\left(t_{1}+s_{n+3}\right)-\cdots-t_{1}
$$

is a path of finite length between $r_{1}+s_{1}$ and $t_{1}$. Hence $N\left(r_{1}+s_{1}\right) \neq \emptyset$, and so $r_{1}+s_{1} \in I$. Now, let $r \in R$. Therefore, $r r_{1}-r r_{2}-\cdots-r r_{n}-r t_{1}$ is a path between $r r_{1}$ and $r t_{1}$ of finite length, and so $N(r x) \neq \emptyset$. Thus $I$ is an ideal of $R$ and $Z(R) \subset I$. It is easy to see that $T(\Gamma(I))$ is a connected subgraph of $T(\Gamma(R))$ containing $Z(\Gamma(R))$. Hence, $Z(\Gamma(R))$ is not disjoint from $\operatorname{Reg}(\Gamma(R))$.

Compare the next theorem with [2, Theorem 2.2].

Theorem 4.8. Let $R$ be a commutative semiring $R$ and $|Z(R)|=\alpha$. Then The following hold:

(i) If $Z(R)$ is a $k$-ideal of $R$ and $2 \in Z(R)$, then $\operatorname{Reg}(\Gamma(R))$ is the union of disjoint complete subgraphs.

(ii) If $Z(R)$ is a $k$-ideal of $R$ and $2 \notin Z(R)$, then $\operatorname{Reg}(\Gamma(R))$ is the union of totally disconnected subgraphs and some connected subgraphs. 
(iii) If $Z(R)$ is a $Q$-ideal of $R,|Q-Z(R)|=\beta$ and $2 \in Z(R)$, then $\operatorname{Reg}(\Gamma(R))$ is the union of $\beta$ disjoint $K^{\lambda}$ 's such that $\lambda \leq \alpha$.

(iv) If $Z(R)$ is a $Q$-ideal of $R,|Q-Z(R)|=\beta$ and $2 \notin Z(R)$, then $\operatorname{Reg}(\Gamma(R))$ is the union of totally disconnected subgraphs and complete bipartite subgraphs.

Proof. (i) Let $2 \in Z(R)$. We set up an equivalence relation $\sim$ on $\operatorname{Reg}(R)$ as follows: for $r, r^{\prime} \in \operatorname{Reg}(R)$, we write $r \sim r^{\prime}$ if and only if $r+r^{\prime} \in Z(R)$. It is straightforward to check that $\sim$ is an equivalence relation on $\operatorname{Reg}(R)$ : for $r \in \operatorname{Reg}(R)$, we denote the equivalence class which contains $r$ by $[r]$. Now let $r \in \operatorname{Reg}(R)$. If $[r]=\{r\}$, then $(r+a)+(r+b)=2 r+(a+b) \in Z(R)$ for every $a, b \in Z(R)$ by Proposition 3.1. So $r+Z(R)$ is a complete subgraph with at most $\alpha$ vertices. If $|[r]|=\gamma>1$, then for every $r^{\prime} \in[r]$ we have $(r+a)+\left(r^{\prime}+b\right)=\left(r+r^{\prime}\right)+a+b \in Z(R)$, where $a, b \in Z(R)$. Thus $r+Z(R)$ is a part of a complete graph $K^{\nu}$ with $\nu \leq \alpha \gamma$ vertices. Therefore, $\operatorname{Reg}(\Gamma(R))$ is the union of disjoint complete subgraphs.

(ii) Let $2 \notin Z(R)$ and $r \in \operatorname{Reg}(R)$. Set

$$
N(r)=\left\{r^{\prime} \in \operatorname{Reg}(R): r+r^{\prime} \in Z(R)\right\} .
$$

If $N(r)=\emptyset$, then $r+r^{\prime} \notin Z(R)$ for every $r^{\prime} \in \operatorname{Reg}(R)$. In this case, we show that $r+Z(R)$ is a totally disconnected subgraph of $\operatorname{Reg}(\Gamma(R))$. If $(r+a)+$ $(r+b) \in Z(R)$ for some $a, b \in Z(R)$, then $2 r+a+b \in Z(R)$; so $2 r \in Z(R)$, which is a contradiction by Proposition 3.1. Therefore, $r+Z(R)$ is a totally disconnected subgraph of $\operatorname{Reg}(\Gamma(R))$. We may assume that $N(r) \neq \emptyset$. Then $r+r^{\prime} \in Z(R)$ for some $r^{\prime} \in \operatorname{Reg}(R)$. Thus $(r+a)+\left(r^{\prime}+b\right)=\left(r+r^{\prime}\right)+(a+b) \in$ $Z(R)$ for every $a, b \in Z(R)$; hence each element of $r+Z(R)$ is adjacent to each element of $r^{\prime}+Z(R)$. If $|N(r)|=\nu$, then we have a connected subgraph of $\operatorname{Reg}(\Gamma(R))$ with at most $\alpha \nu$ vertices. Hence, If $2 \notin Z(R)$, then $\operatorname{Reg}(\Gamma(R))$ is the union of totally disconnected subgraphs and some connected subgraphs.

(iii) First, we show that $q+Z(R) \subseteq \operatorname{Reg}(R)$ for every $q \in Q-Z(R)$. If $q+a \notin \operatorname{Reg}(R)$ for some $a \in Z(R)$, then $q+a \in Z(R)$; hence $q \in Z(R)$ since $Z(R)$ is a k-ideal which is a contradiction. Let $2 \in Z(R)$ and $q \in Q-Z(R)$. Then each coset $q+Z(R)$ is a complete subgraph of $\operatorname{Reg}(R)$ with $\lambda$ vertices such that $\lambda \leq \alpha$ (note that $\left(q_{1}+Z(R)\right) \cap\left(q_{2}+Z(R)\right) \neq \emptyset$ if and only if $\left.q_{1}=q_{2}\right)$ since $(q+a)+(q+b)=2 q+(a+b) \in Z(R)$ for all $a, b \in Z(R)$ by Proposition 3.1 and $Z(R)$ is an ideal. Next, we show that distinct cosets form disjoint subgraphs of $\operatorname{Reg}(\Gamma(R))$. If $q_{1}+a$ and $q_{2}+b$ are adjacent for some $q_{1}, q_{2} \in Q-Z(R)$ and $a, b \in Z(R)$, then $\left(q_{1}+a\right)+\left(q_{2}+b\right) \in Z(R)$ gives $q_{1}+q_{2} \in Z(R)$ since $Z(R)$ is a $k$-ideal of $R$. So $q_{2}+2 q_{1}=q_{1}+\left(q_{1}+q_{2}\right) \in q_{1}+Z(R)$. Likewise, $q_{2}+2 q_{1} \in q_{2}+Z(R)$ by Proposition 3.1. So $q_{2}+2 q_{1} \in\left(q_{1}+Z(R)\right) \cap\left(q_{2}+Z(R)\right)$; hence $q_{1}=q_{2}$. Thus $\operatorname{Reg}(\Gamma(R))$ is the union of $\beta$ disjoint induced subgraphs $q+Z(R)$, each of which is a $K^{\lambda}$ such that $\lambda \leq \alpha$. 
(iv) Assume that $2 \notin Z(R)$ and let $q \in Q-Z(R)$. If $q+q^{\prime} \notin Z(R)$ for every $q^{\prime} \in Q-Z(R)$, then $N(q)=\emptyset$. Then by (ii), $q+Z(R)$ is a totally disconnected subgraph of $\operatorname{Reg}(\Gamma(R))$. So we may assume that $q+q^{\prime} \in Z(R)$ for some $q^{\prime} \in$ $Q-Z(R)$. Then by (ii) each element of $q+Z(R)$ is adjacent to each element of $q^{\prime}+Z(R)$. Now we show that $q^{\prime}$ is the unique element. Let $q+q^{\prime \prime} \in Z(R)$ for some $q^{\prime \prime} \in Q-Z(R)$. Therefore, $q+q^{\prime}+q^{\prime \prime}=q^{\prime}+\left(q+q^{\prime \prime}\right) \in q^{\prime}+Z(R)$. Likewise, $q+q^{\prime}+q^{\prime \prime}=q^{\prime \prime}+\left(q+q^{\prime}\right) \in q^{\prime \prime}+Z(r)$. Thus $\left(q^{\prime}+Z(R)\right) \cap\left(q^{\prime \prime}+z(R)\right) \neq \emptyset$ gives $q^{\prime}=q^{\prime \prime}$. Therefore $(q+Z(R)) \cup\left(q^{\prime}+Z(R)\right)$ is a complete bipartite subgraph of $\operatorname{Reg}(\Gamma(R))$. So $\operatorname{Reg}(\Gamma(R))$ is the union of totally disconnected subgraphs and complete bipartite subgraphs.

Proposition 4.9. Let $R$ be a commutative semiring $R$. Then the following hold:

(i) If $Z(R)$ is a $k$-ideal of $R$ and $\operatorname{Reg}(\Gamma(R))$ is complete, then $|\operatorname{Reg}(R)|=1$ or $|\operatorname{Reg}(R)|=2$.

(ii) If $Z(R)$ is a $Q$-ideal of $R$ and $\operatorname{Reg}(\Gamma(R))$ is complete, then $|R / Z(R)|=2$ or $|R / Z(R)|=3$.

(iii) If $Z(R)$ is a $Q$-ideal of $R,|R / Z(R)|=2$ and $2 \in Z(R)$, then $\operatorname{Reg}(\Gamma(R))$ is complete.

Proof. (i) If $2 \in Z(R)$, then $2 r \in Z(R)$ for every $r \in \operatorname{Reg}(R)$. Then $r+Z(R)$ is a complete subgraph of $\operatorname{Reg}(\Gamma(R))$; hence $|\operatorname{Reg}(R)|=1$ since $\operatorname{Reg}(\Gamma(R))$ is complete. If $2 \notin Z(R)$, then for each $r \in \operatorname{Reg}(R)$, there exists $r^{\prime} \in \operatorname{Reg}(R)$ such that $r+r^{\prime} \in Z(R)$. So $|\operatorname{Reg}(R)|=2$ since $\operatorname{Reg}(\Gamma(R))$ is complete. In this case, $\operatorname{Reg}(\Gamma(R))$ is a complete bipartite graph (see Theorem 4.8).

(ii) Since every $Q$-ideal is a $k$-ideal, the part (i) gives $|\operatorname{Reg}(R)|=1$ or $|\operatorname{Reg}(R)|=2$. If $|\operatorname{Reg}(R)|=1$, then $R=Z(R) \cup(q+Z(R))$ for $q \in \operatorname{Reg}(R)$ and hence $|R / Z(R)|=2$. Similarly, if $|\operatorname{Reg}(R)|=2$, then $R=Z(R) \cup(q+$ $Z(R)) \cup\left(q^{\prime}+Z(R)\right)$ for $q, q^{\prime} \in \operatorname{Reg}(R)$ with $q \neq q^{\prime}$, and hence $|R / Z(R)|=3$.

(iii) By assumption, $R=Z(R) \cup(q+Z(R))$ for some $q \in Q-Z(R)$; so $2 q \in Z(R)$ by Proposition 3.1. Let $r, r^{\prime} \in \operatorname{Reg}(R)$. Then $r, r^{\prime} \in q+Z(R)$. So $\left.r+r^{\prime}=(q+a)+(q+b)=2 q+(a+b) \in Z(R)\right)$ for some $a, b \in Z(R)$. Thus $\operatorname{Reg}(\Gamma(M))$ is complete.

Proposition 4.10. Let $R$ be a commutative semiring such that $Z(R)$ is a $Q$-ideal of $R$. Then the following hold:

(i) If $\operatorname{Reg}(\Gamma(R))$ is connected, then $|R / Z(R)|=2$ or $|R / Z(R)|=3$.

(ii) If $|R / Z(R)|=2$ and $2 \in Z(R)$, then $\operatorname{Reg}(\Gamma(R))$ is connected.

Proof. (i) Let $\operatorname{Reg}(\Gamma(R))$ be a connected graph. Then $\operatorname{Reg}(\Gamma(R))$ is a single complete graph $K^{\lambda}$ or a bipartite graph by Theorem 4.8. Hence $\operatorname{Reg}(\Gamma(R))$ is a complete graph. Now the assertion follows from Proposition 4.9.

(ii) This follows directly from Proposition 4.9. 
Theorem 4.11 Let $R$ be a commutative semiring $R$. Then the following hold:

(i) If $Z(R)$ is a $k$-ideal of $R$, then $\operatorname{diam}(\operatorname{Reg}(\Gamma(R)))=0$ if and only if $Z(R)=\{0\}$ and $|R|=2$.

(ii) Let $Z(R)$ be a $Q$-ideal of $R$. Then:

(a) $\operatorname{diam}(\operatorname{Reg}(\Gamma(R)))=1$ if and only if $2 \in Z(R)$ and $|R / Z(R)|=2$.

(b) $\operatorname{diam}(\operatorname{Reg}(\Gamma(R)))=2$ if and only if $|R / Z(R)|=3,2 \notin Z(R)$ and $q+q^{\prime} \in Z(R)$ for every $q, q^{\prime} \in Q-Z(R)$.

(c) Otherwise $\operatorname{diam}(\operatorname{Reg}(\Gamma(R)))=\infty$.

Proof. (i) If $\operatorname{diam}(\operatorname{Reg}(\Gamma(R)))=0$, then $\operatorname{Reg}(\Gamma(R))$ is a complete graph $K^{1}$, and so $|Z(R)|=|\operatorname{Reg}(R)|=1$ by Theorem 4.8. Hence $Z(R)=\{0\}$ and $|R|=2$. The other implication is clear.

(ii) (a) If $\operatorname{diam}(\operatorname{Reg}(\Gamma(R)))=1$, then $\operatorname{Reg}(\Gamma(R))$ is a complete graph $K^{\lambda}$ with $\lambda \leq|Z(R)|$ by Theorem 4.8. Therefore, $2 \in Z(R)$ and $|Q-Z(R)|=1$. Thus $R=Z(R) \cup(q+Z(R))$ for some $q \in Q-Z(R)$; hence $|R / Z(R)|=2$. The converse follows from Theorem 4.8 .

(ii) (b) If $\operatorname{diam}(\operatorname{Reg}(\Gamma(R)))=2$, then $\operatorname{Reg}(\Gamma(R))$ is a complete bipartite graph $K^{1,2}$ or $K^{2,2}$; thus $2 \notin Z(R)$ and $|Q-Z(R)|=2$ by Theorem 4.8. Since $\operatorname{Reg}(\Gamma(R))$ has not any totally disconnected subgraph, we must have $q+q^{\prime} \in Z(R)$ for every $q, q^{\prime} \in Q-Z(R)$.

Remark 4.12. Let $R$ and $M$ be as described in Example 4.5. So $Z(R)=$ $\{0,2,4,6, \cdots\}$ is a $k$-ideal of $R$ but it is not a $Q$-ideal of $R$. Also, $Z(\Gamma(R))$ is a complete graph and $\operatorname{Reg}(\Gamma(R))$ is a totally disconnected graph. Since $\operatorname{gcd}(2,4)=2$, we have $2 * 4=0$; hence $2 \in Z(R)$. Moreover, $R=Z(R) \cup(1+$ $Z(R))$ and $\operatorname{diam}(\operatorname{Reg}(\Gamma(R)))=\infty$. Hence Theorem 4.11 (ii) is not true when $Z(R)$ is not a $Q$-ideal of $R$.

Proposition 4.13. Let $R$ be a commutative semiring such that $Z(R)$ is a $k$-ideal of $R$. Then $\operatorname{gr}(\operatorname{Reg}(\Gamma(R)))=3,4$ or $\infty$. In particular, if $\operatorname{Reg}(\Gamma(R))$ contains a cycle, $\operatorname{gr}(\operatorname{Reg}(\Gamma(R))) \leq 4$.

Proof. Let $\operatorname{Reg}(\Gamma(R))$ contains a cycle. Then $\operatorname{Reg}(\Gamma(R))$ is not a totally disconnected graph, so by the proof of Theorem $4.8, \operatorname{Reg}(\Gamma(R))$ has either a complete or a complete bipartite subgraph. Therefore, it must contain either a 3-cycle or a 4-cycle. Thus $\operatorname{gr}(\operatorname{Reg}(\Gamma(R))) \leq 4$.

Theorem 4.14. Let $R$ be a commutative semiring such that $Z(R)$ be a $k$-ideal of $R$. Then the following hold:

(i) $\operatorname{gr}(\operatorname{Reg}(\Gamma(R)))=3$ if and only if $2 \in Z(R)$ and $|r+Z(R)| \geq 3$ for some $r \in \operatorname{Reg}(R)$.

(ii) $\operatorname{gr}(\operatorname{Reg}(\Gamma(R)))=4$ if and only if $2 \notin Z(R)$ and $r+r^{\prime} \in Z(R)$ for some $r, r^{\prime} \in \operatorname{Reg}(R)$. 
Proof. (i) Assume that $\operatorname{gr}(\operatorname{Reg}(\Gamma(R)))=3$. Then by Theorem 4.8, $\operatorname{Reg}(\Gamma(R))$ is a complete graph $K^{\lambda}$ with $3 \leq \lambda$. Therefore, $2 \in Z(R)$ and $|r+Z(R)| \geq 3$ for some $r \in \operatorname{Reg}(R)$.

(ii) If $\operatorname{gr}(\operatorname{Reg}(\Gamma(R)))=4$, then by Theorem 4.8, $\operatorname{Reg}(\Gamma(R))$ has a complete bipartite subgraph; hence $2 \notin Z(R)$ and $r+r^{\prime} \in Z(R)$ for some $r, r^{\prime} \in \operatorname{Reg}(R)$ by Theorem 4.8. The other implications of (i) and (ii) follows directly from Theorem 4.8.

Theorem 4.15. Let $R$ be a commutative semiring such that $Z(R)$ be a $k$-ideal of $R$. Then the following hold:

(i) $\operatorname{gr}(T(\Gamma(R)))=3$ if and only if $|Z(R)| \geq 3$.

(ii) $\operatorname{gr}(T(\Gamma(R)))=4$ if and only if $2 \notin Z(R),|Z(R)|<3$ and $r+r^{\prime} \in Z(R)$ for some $r, r^{\prime} \in \operatorname{Reg}(R)$.

(iii) Otherwise, $\operatorname{gr}(T(\Gamma(R)))=\infty$.

Proof. (i) This follows from Proposition 4.1.

(ii) Since $\operatorname{gr}(Z(\Gamma(R))=3$ or $\infty$, then $\operatorname{gr}(\operatorname{Reg}(\Gamma(R)))=4$. Therefore, $2 \notin Z(R)$ and $r+r^{\prime} \in Z(R)$ for some $r, r^{\prime} \in \operatorname{Reg}(R)$ by Theorem 4.14. On the other hand, $\operatorname{gr}(T(\Gamma(R)) \neq 3$; so $|Z(R)|<3$. The other implication follows from Theorem 4.8 .

Acknowledgements The authors are very grateful to the referee for suggesting ways to make this paper read better.

\section{References}

[1] D. D. Anderson and M. Naseer, Beck's coloring of a commutative ring, J. Algebra, 159 (1993), 500-514.

[2] D. F. Anderson and A. Badawi, The total graph of a commutative ring, J. Algebra, 320 (2008), 2706-2719.

[3] D. F. Anderson and P. F. Livingston, The zero-divisor graph of a commutative ring, J. Algebra, 217 (1999), 437-447.

[4] P. J. Allen, A fundamental theorem of homomorphisms for semirings, Proc. Amer. Math. Soc. 21 (1969), 412-416.

[5] I. Beck, Coloring of a commutative ring, J. Algebra, 116 (1988), 208-226.

[6] S. Ebrahimi Atani, The ideal theory in quotients of commutative semirings, Glas. Math., 42 (2007), 301-308. 
[7] S. Ebrahimi Atani, The zero-divisor graph with respect to ideals of a commutative semiring, Glas. Math., 43 (2008), 309-320.

[8] S. Ebrahimi Atani, An ideal-based zero-divisor graph of a commutative semiring, Glas. Math., 44 (1) (2009), 141-153.

[9] S. Ebrahimi Atani and R. Ebrahimi Atani, Some remarks on partitioning semirings, An. St. Univ. Ovidius Constanta, 18(1) (2010), 49-62.

[10] S. Ebrahimi Atani and S. Habibi, The total torsion element graph of a module over a commutative ring, An. St. Univ. Ovidius Constanta, 19(1) (2011), 23-34.

[11] J. S. Golan, The theory of semirings with applications in mathematics and theoretical computer Science, Pitman Monographs and Surveys in Pure and Applied Mathematics, Longman Scientific and Technical, Harlow UK, (1992).

[12] J. S. Golan, Semirings and their Applications, Kluwer Academic Publishers, Dordrecht, (1999).

[13] S. B. Mulay, Cycles and symmetries of zero-divisors, Comm. Algebra, 30(7) (2002), 3533-3558.

Shahabaddin Ebrahimi Atani,

Faculty of Mathematical Sciences,

University of Guilan,

P.O. Box 1914,Rasht, Iran.

Email: ebrahimi@guilan.ac.ir

Fatemeh Esmaeili Khalil Saraei,

Faculty of Mathematical Sciences,

University of Guilan,

P.O. Box 1914,Rasht, Iran.

Email: f.esmaeili.kh@gmail.com 